\title{
On the building blocks of the M31 and Milky Way halos
}

\author{
Matteo Monelli ${ }^{1,2, \star}$ \\ ${ }^{1}$ Instituto de Astrofísica de Canarias (IAC), E-38205 La Laguna, Tenerife, Spain \\ ${ }^{2}$ Universidad de La Laguna (ULL), Dpto. Astrofísica, E-38206 La Laguna, Tenerife, Spain
}

\begin{abstract}
We discuss the formation of the halo of M31 and the Milky Way as traced by the population of RR Lyrae stars, in comparison with the population of such stars preent in satellite dwarf galaxies. We find that both halos and the massive dwarf host a population of high amplitude short period RRab stars, absent in low-mass dwarfs. These stars are explained as the metal-rich tail of the RR Lyrae distribution $([\mathrm{Fe} / \mathrm{H}] \sim-1.5)$, and thus their existence imply fast chemical enrichment in the host system. Their presence in both halos implies that massive building blocks had an important rôle in their formation.
\end{abstract}

\section{Introduction}

RR Lyrae stars (RRL) are excellent tracers of the old population, and bring direct insights on the environmental conditions of the host stellar systems at early epochs. For this reason, they are important tracers of the mass accretion history of the halo of spiral galaxies such as M31 and the Milky Way (MW). [6] presented a comparison of the properties of RRL stars in the MW halo and in its system of globular clusters and satellite dwarfs. In particular, they detected the existence of a family or RRab type stars characterized by high amplitudes and short periods $\left(P<0.48 \mathrm{~d}, A_{V}>0.75 \mathrm{mag}\right)$, hence the name, HASP). These stars are associated to a population of stars as metal-rich as $[\mathrm{Fe} / \mathrm{H}] \sim-1.5$. [6] discusses the existence of such stars in massive MW satellites such as the LMC and the Sagittarius $\mathrm{dSph}$, in metal-rich globular clusters but not in lower mass galaxies. This indicates that $i$ ) low-mass dwarfs did not experience a chemical evolution fast enough to produce HASP, and ii) to explain the existence of HASP in the MW halo one has to accept that massive building blocks, already enriched at the time of accretion, had to participate in the halo construction.

\section{On the M31 halo building blocks}

We have performed a similar analysis in the case of M31. Figure 1 compares the period distributions of RRL stars in the M31 and MW halos, and with their satellite systems, as detailed in the caption. The plot shows that the normalized period distribution of both halos is very similar to the corresponding sample of bright satellites. The histograms relative to the RRL stars from faint galaxies are charaterized by a rising branch and the main peak occurring at longer period. This reflects the absence of HASP stars in these systems. The plot clearly shows that to repodure the period distribution of both

${ }^{\star}$ monelli@iac.es 


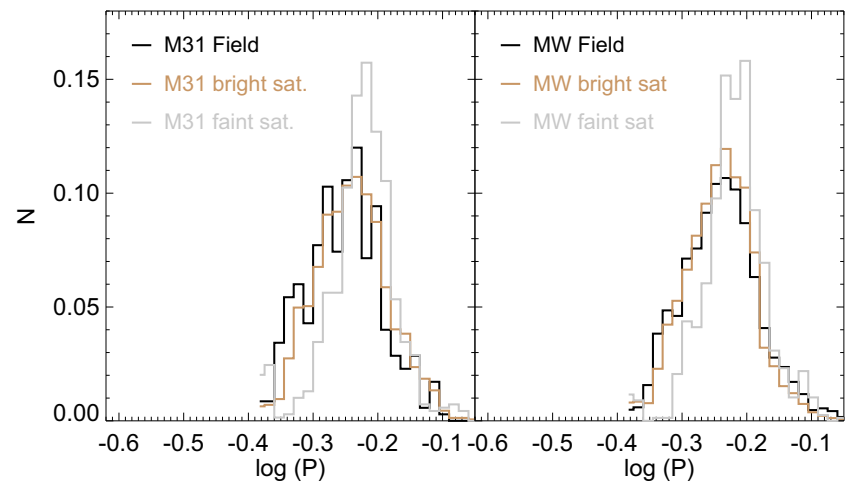

Figure 1. Period distribution of RRab star in different samples of galaxies. The adopted data are M31 halo: [7] and Field 2 from [11]. $M W$ halo: same collection as in [6]. M31 bright satellites: dwarfs with $M_{V}<-12$, namely: And VII, NGC 147, and NGC 185 from Monelli et al. (2017, submitted); M32: [5]; And II, from the compilation of Martínez-Vázquez et al. (2017, submitted). MW bright satellites: OGLE-III data for the LMC, the SMC and Sagittarius dwarf spheroidal ([13-15]); M31 faint satellites: And I, And III, And XV, And XVI and And XXVIII from Martínez-Vázquez et al. (2017, op. cit.); And VI ([10]); And XI and And XIII ([18]); And XIX ([2]), And XXI ([3]) and And XXV ([4]) observed with the Large Binocular Telescope; faint MW satellites: Carina ([1]); Leo II ([12]); Draco ([8]); Sculptor ([9]); Leo I ([17]).

the MW and the M31 halo the contribution of massive dwarf galaxies is required. A KolomogorovSmirnov test performed on the period distributions supports this conclusion, as the probability that the the M31 (MW) halo distribution is drawn from the same one as the bright satellite's is $46 \%$ (99\%).

\section{References}

[1] Coppola, G., Marconi, M., Stetson, P. B., et al., ApJ, 814, 71 (2015)

[2] Cusano, F., Clementini, G., Garofalo, A., et al., ApJ, 779, 7 (2013)

[3] Cusano, F., Garofalo, A., Clementini, G., et al., ApJ, 806, 200 (2015)

[4] Cusano, F., Garofalo, A., Clementini, G., et al., ApJ, 829, 26 (2016)

[5] Fiorentino, G., Contreras Ramos, R., Tolstoy, E., et al., A\&A, 539, A138 (2012)

[6] Fiorentino, G., Bono, G., Monelli, M., et al., ApJL, 798, L12 (2015)

[7] Jeffery, E. J., Smith, E., Brown, T. M., et al., AJ, 141, 171 (2011)

[8] Kinemuchi, K., Harris, H. C., Smith, H. A., et al., AJ, 136, 1921 (2008)

[9] Martínez-Vázquez, C. E., Monelli, M., Bono, G., et al., MNRAS, 454, 1509 (2015)

[10] Pritzl, B. J., Armandroff, T. E., Jacoby, G. H., \& Da Costa, G. S., AJ, 124, 1464 (2002)

[11] Sarajedini, A., Mancone, C. L., Lauer, T. R., et al., AJ, 138, 184 (2009)

[12] Siegel, M. H., \& Majewski, S. R., AJ, 120, 284 (2000)

[13] Soszyński, I., Udalski, A., Szymański, M. K., et al., AcA, 60, 165 (2010)

[14] Soszyński, I., Udalski, A., Szymański, M. K., et al., AcA, 59, 1 (2009)

[15] Soszyński, I., Dziembowski, W. A., Udalski, A., et al., AcA, 61, 1 (2011)

[16] Soszyński, I., Udalski, A., Szymański, M. K., et al., AcA, 65, 233 (2015)

[17] Stetson, P. B., Fiorentino, G., Bono, G., et al., PASP, 126, 616 (2014)

[18] Yang, S.-C., Wagner-Kaiser, R., Sarajedini, A., Kim, S. C., \& Kyeong, J., ApJ, 784, 76 (2014) 